\section{(2) OPEN ACCESS}

\title{
Computed tomographic myocardial mass compared with invasive myocardial perfusion measurement
}

\author{
Daniëlle C J Keulards (D) ,' Stephane Fournier, ${ }^{2,3}$ Marcel van 't Veer, ${ }^{1,4}$ Iginio Colaiori, ${ }^{3}$ \\ Jo M Zelis (D) , ${ }^{1}$ Mohamed El Farissi, ${ }^{1}$ Frederik M Zimmermann, ${ }^{1}$ Carlos Collet, ${ }^{3}$ \\ Bernard De Bruyne, 2,3 Nico H J Pijls ${ }^{1,4}$
}

- Additional material is published online only. To view please visit the journal online (http://dx.doi.org/10.1136/ heartjil-2020-316689).

${ }^{1}$ Department of Cardiology, Catharina Hospital, Eindhoven, North Brabant, The Netherlands ${ }^{2}$ Department of Cardiology, University Hospital of Lausanne, Lausanne, Switzerland ${ }^{3}$ Department of Cardiology, Cardiovascular Center Aalst, OLV Clinic Aalst, Leopoldlaan, Belgium

${ }^{4}$ Department of Biomedical Engineering, Eindhoven University of Technology, Eindhoven, The Netherlands

Correspondence to Dr Daniëlle C J Keulards, Department of Cardiology, Catharina Hospital, Eindhoven 5623 EJ, North Brabant, The Netherlands; danielle.keulards@ catharinaziekenhuis.n

DCJK and SF contributed equally.

Received 12 February 2020 Revised 23 April 2020 Accepted 24 April 2020 Published Online First 29 May 2020

\section{Linked}

http://dx.doi.org/10.1136/ heartjnl-2020-317155

\section{Check for updates}

(C) Author(s) (or their employer(s)) 2020. Re-use permitted under CC BY-NC. No commercial re-use. See rights and permissions. Published by BMJ.

To cite: Keulards DCJ,

Fournier $S$, van 't

Veer $\mathrm{M}$, et al. Heart

2020;106:1489-1494.

\section{ABSTRACT}

Objective The prognostic importance of a coronary stenosis depends on its functional severity and its depending myocardial mass. Functional severity can be assessed by fractional flow reserve (FFR), estimated non-invasively by a specific validated CT algorithm $\left(F_{C T}\right)$. Calculation of myocardial mass at risk by that same set of CT data (CTmass), however, has not been prospectively validated so far. The aim of the present study was to compare relative territorial-based CTmass assessment with relative flow distribution, which is closely linked to true myocardial mass.

Methods In this exploratory study, 35 patients with (near) normal coronary arteries underwent CT scanning for computed flow-based CTmass assessment and underwent invasive myocardial perfusion measurement in all 3 major coronary arteries by continuous thermodilution. Next, the mass and flows were calculated as relative percentages of total mass and perfusion.

Results The mean difference between CTmass per territory and invasively measured myocardial perfusion, both expressed as percentage of total mass and perfusion, was $5.3 \pm 6.2 \%$ for the left anterior descending territory, $-2.0 \pm 7.4 \%$ for the left circumflex territory and $-3.2 \pm 3.4 \%$ for the right coronary artery territory. The intraclass correlation between the two techniques was 0.90 .

Conclusions Our study shows a close relationship between the relative mass of the perfusion territory calculated by the specific CT algorithm and invasively measured myocardial perfusion. As such, these data support the use of CTmass to estimate territorial myocardium-at-risk in proximal coronary arteries.

\section{INTRODUCTION}

In patients with coronary artery disease, the most important parameters for both symptoms and outcome are the presence and extent of inducible ischaemia. ${ }^{1-4}$

Fractional flow reserve (FFR) measurement during cardiac catheterisation is the invasive standard for the presence of ischaemia and is useful to determine if a patient benefits from percutaneous coronary intervention. An FFR $\leq 0.80$ indicates functional significance of a particular stenosis. Because FFR is determined by the stenosis itself and also by the amount of viable myocardium distal to that stenosis, the same FFR value may have a different prognostic significance depending on the extent of the distal myocardium. It is known from previous trials that patients benefit most from revascularisation if FFR is $\leq 0.80$ or if at least $10 \%-12.5 \%$ of the myocardium is at ischaemic risk. ${ }^{12}$ Therefore, information about the extent of the perfusion territory distal to a specific coronary stenosis, in addition to FFR of that stenosis, is clinically relevant. ${ }^{34}$

Up to now, no method has been validated to assess the amount of myocardium at risk in conjunction with simultaneous assessment of functional stenosis severity. Both MRI and CT scanning are able to estimate myocardial mass non-invasively, but no stenosis-specific functional information can be obtained. ${ }^{5} 6$ During the last decade, a method to compute FFR from coronary CT-angiographic images along the coronary tree has been developed and validated and is known as $\mathrm{FFR}_{\mathrm{CT}}{ }^{7-9}$ (HeartFlow, Redwood City, CA, USA). Several studies have investigated coronary volume to myocardial (V/M) mass computations using $\mathrm{FFR}_{\mathrm{CT}}$, but calculation of relative mass per territory (expressed as percentage of total mass) has not been reported. ${ }^{10-13}$

Experimentally, it has been shown that an excellent correlation exists between coronary blood flow and mass of the perfused territory. ${ }^{14-16}$ Therefore, direct measurement of myocardial perfusion is a good approach to validate myocardial mass.

The purpose of this exploratory study was to compare the relative myocardial mass distribution assessed by that specific CT algorithm to the respective distribution of blood flow measured invasively in the three major coronary arteries. If such relation can be demonstrated, both the functional significance of the stenosis $\left(\mathrm{FFR}_{\mathrm{CT}}\right)$ and its depending myocardial mass (CTmass) can be assessed noninvasively by one single CT examination.

\section{METHODS}

\section{Design and study population}

This study was performed in 35 patients with normal coronary arteries or minimal atherosclerosis between July 2017 and December 2018. These patients were characterised by angiographically normal coronary arteries (ie, no signs of atherosclerosis on angiogram or less than a 30\% lumen reduction in one segment only; non-ischaemic FFR values (ie, $>0.80$ ); no lumen reductions of more than $30 \%$ in more than one segment on the CT scan). An invasive angiogram in these patients was performed 
because of persistent non-specific thoracic complaints. Patients with coronary anomalies, valvular disease, known left ventricular hypertrophy or pulmonary hypertension were excluded. Other exclusion criteria included contraindications for CT scanning and age above 75 . The coronary CT angiogram (CTA) and invasive angiography were performed less than 3 months apart without interventions in between.

\section{Patient and public involvement statement}

Patients were screened and signed informed consent before all examinations. This research was done without patient involvement.

\section{CT scanning for calculation of myocardial mass}

All 35 patients underwent a coronary CTA performed on a 256slice scanner (Brilliance iCT, Philips Healthcare or SOMATOM, Siemens). The coronary CTA scan parameters were as follows: tube potential, $120 \mathrm{kVp}$; field of view, $\leq 255 \mathrm{~mm}$; slice thickness, $0.9 \mathrm{~mm}$; increment, $0.45 \mathrm{~mm}$. Prior to CT examination, patients were pretreated with $0.8 \mathrm{mg}$ sublingual nitroglycerine and intravenous metoprolol if the heart rate was $\geq 65$ beats $/ \mathrm{min}$. Thereafter, ECG-gated step-and-shoot images (in two stacks at $75 \%$ of the heart cycle) were made. The scan procedure included a non-contrast ECG-triggered coronary artery calcium scoring scan directly followed by a diagnostic coronary CTA scan using iomeron 300 with a flow of at least $6 \mathrm{~mL} / \mathrm{s}$. Coronary CTA images were transferred to a core laboratory at HeartFlow Inc. (Redwood City, CA, USA), and the images were screened for interpretability by detecting image artefacts automatically. First, all images were processed through deep learning-based centreline and lumen extraction algorithms and checked for anatomical correctness of the segmented geometry and the left ventricular myocardium. ${ }^{9} 17$ The left ventricle (LV) was segmented using standard methods and the LV myocardial mass was computed by multiplying the extracted myocardial volume by a tissue density of $1.05 \mathrm{~g} / \mathrm{cc}$. For the relative mass calculation, the coronary centreline trees were traversed and the centrelines of the main branches in the vicinity of the segmented LV myocardium were classified as perfusing the LV using a distance threshold based on vessel size. ${ }^{18}$ Vessels that were identified to feed other heart chambers (right ventricle (RV)) were classified as non-LV feeding vessels. Next, the total baseline flow to the LV feeding vessels was computed using the LV myocardial mass and an assumed flow per unit tissue as previously described. ${ }^{9}$ The total baseline flow through the non-LV feeding vessels was estimated to be $20 \%$ of LV baseline flow based on literature data. ${ }^{19} \mathrm{Next}$, total flow to the LCA and RCA was distributed to the individual vessels within these territories based on downstream vascular volume. Finally, the myocardial mass subtended by each coronary vessel territory (left anterior descending (LAD), left circumflex (LCX) and right coronary artery (RCA)) was computed by dividing the territory flow (calculated as the product of the coronary artery cross-sectional area and area averaged velocity) by a constant baseline flow per unit tissue and the fraction of total myocardial mass was reported. All corelab personnel were blinded.

\section{Invasive measurement of absolute coronary blood flow}

Cardiac catheterisation with FFR and absolute blood flow measurements was performed according to routine by either the femoral or the radial access, at the discretion of the operator. Guiding catheters were advanced into the coronary arteries and after intracoronary administration of $200 \mu \mathrm{g}$ nitroglycerin and proper equalisation of pressures, FFR was measured in all three

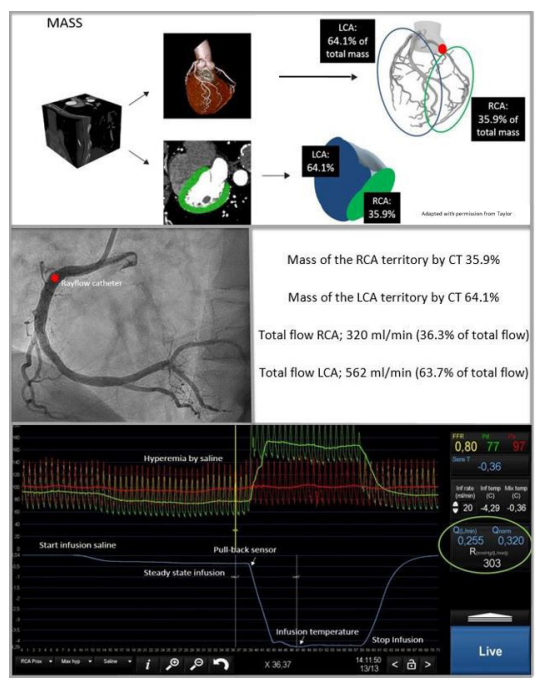

Figure 1 Left upper panel shows the CT angiogram images derived by the physiological CT algorithm (HeartFlow). Dedicated software was used to calculate mass distal to the position of the Rayflow catheter. All patients underwent coronary angiography (middle left panel) with invasive blood flow and resistance measurements in all three coronary arteries (bottom). ${ }^{10}$ LCA, left carotid artery; RCA, right carotid artery.

major coronary arteries using a pressure/temperature wire (PressureWire X; Abbott, Saint Paul, MN, USA); following, a dedicated monorail infusion catheter (Rayflow; Hexacath, Paris) was advanced over the pressure wire and positioned with its tip in the proximal part of the coronary artery. The pressure/temperature sensor of the pressure wire was positioned in the distal part of the coronary artery.

Next, saline infusion was started at a rate of $20 \mathrm{~mL} / \mathrm{min}$ (Qi) and absolute blood flow in the coronary artery was calculated as previously described. ${ }^{20-22}$

In short, this method provides calculation of hyperemic coronary blood flow $(\mathrm{mL} / \mathrm{min})$. During continuous infusion of saline at a set rate $(\mathrm{Qi}, \mathrm{mL} / \mathrm{min})$, the temperature of the completely mixed blood and saline (T) is measured in the distal coronary artery and after a steady-state has been reached (within 10-20 s), the pressure wire is pulled back in the Rayflow catheter to determine the infusion temperature of the saline (Ti). Absolute blood flow $(\mathrm{mL} / \mathrm{min})$ is calculated by the equation

$$
Q=1.08 \frac{T i}{T} x Q i
$$

The constant 1.08 relates to the difference between the specific heats and densities of blood and saline.

All signals are continuously displayed on the regular cathlab monitor by the Coroventis software (Coroflow, Uppsala, Sweden; figure 1).

\section{Comparison of CT mass and invasively measured perfusion}

The invasively measured blood flow is the flow distal to the point of the infusion, that is, at the tip of the infusion catheter. To compare these measurements with the non-invasive CT data, the exact position of the Rayflow catheter was taken into account to determine the amount of myocardial mass (figure 1).

Because even in normal coronary arteries, the presence of the infusion catheter may influence the measured blood flow (creating a small gradient), the flow in the absence of a stenosis is determined by the Coroventis software (Qnorm) by dividing the actually measured flow by FFR. 


\begin{tabular}{lc}
\hline Table $1 \quad$ Baseline characteristics & \\
\hline Patients & $\mathrm{n}=35$ \\
\hline Male gender, $\mathrm{N}(\%)$ & $20(57.1)$ \\
\hline Age, mean (SD) & $59.3(9.4)$ \\
\hline Medical history & $9(25.7)$ \\
\hline Hypertension, $\mathrm{n}(\%)$ & $6(17.1)$ \\
\hline Current smoking, $\mathrm{n}(\%)$ & $3(8.6)$ \\
\hline Diabetes mellitus type $2, \mathrm{n}(\%)$ & $17(48.6)$ \\
\hline Dyslipidaemia, $\mathrm{n}(\%)$ & $12(34.3)$ \\
\hline Family history of CAD, $\mathrm{n}(\%)$ & \\
\hline Selection criteria & \\
\hline Angiographic details & $19(54.3)$ \\
\hline Completely normal coronaries & $16(45.7)$ \\
\hline Minimal wall irregularities * & $0.86 \pm 0.06$ \\
\hline FFR LAD ${ }^{*}$ & $0.95 \pm 0.03$ \\
\hline FFR LCX $\dagger$ & $0.94 \pm 0.05$ \\
\hline FFR RCA $\dagger$ & \\
\hline CT details & $35(100)$ \\
\hline Agatston score $<400$ & $207.5 \pm 156.3$ \\
\hline Absolute Agatston score & $0.87 \pm 0.05$ \\
\hline FFR-CT LAD & $0.90 \pm 0.04$ \\
\hline FFR-CT LCX & $0.92 \pm 0.04$ \\
\hline FFR-CT RCA & \\
\hline Sun & \\
\hline
\end{tabular}

Summary values represent number (\%) or mean \pm SD.

* Minimal wall irregularities: patients with wall irregularities $\leq 30 \%$ in one coronary segment only.

tinvasively measured FFR with adenosine and in the absence of the Rayflow catheter.

$C A D$, coronary artery disease; $F F R$, fractional flow reserve; $L A D$, left anterior descending; LCX, left circumflex; RCA, right coronary artery

Assuming homogeneous myocardial perfusion and assuming a direct relation between blood flow and mass of the perfused territory, the relative distributions of both the mass and flow should be equal. Therefore, the relative amount of myocardial mass of the LAD, LCX and RCA artery will be compared with the relative blood flow in the respective coronary arteries. As an example, if the myocardial mass perfused by the LAD equals $37 \%$ of total myocardial mass and flow measured in the LAD equals $40 \%$ of total measured myocardial flow, the percentual difference is $3 \%$.

\section{Statistical analysis}

Analyses were performed using R V.3.5.2 (R Foundation for Statistical Computing, Vienna, Austria). The package 'ggplot2' was used for the graphical plots. The package 'blandr' was used for the Bland Altman and 'psych' for the intraclass correlation (ICC) analyses. The ICC technique used is the two-way mixedeffects model estimated for a single rater. Bland-Altman plots were made per vessel (LAD, LCX and RCA) to compare mass and flow distributions. Mean values and \pm 1.96 SD are plotted. Categorical variables are presented as counts and percentages. Continuous variables are presented as mean $\pm \mathrm{SD}$ as appropriate.

\section{RESULTS}

\section{Patient characteristics and procedural results}

A total of 35 normal patients with a mean age of $59.3 \pm 9.4$ were included, undergoing coronary angiography and flow measurements in all three major coronary arteries and CT scanning. CT scans were sent for analysis; two were rejected due to motion artefacts, and these patients were excluded. Patient characteristics are listed in table 1 . Successful invasive measurements were

\begin{tabular}{|c|c|c|c|}
\hline \multicolumn{4}{|l|}{ Variables } \\
\hline Vessel measured & LAD & LCX & $\mathrm{RCA}$ \\
\hline & $35(100)$ & $35(100)$ & $35(100)$ \\
\hline Myocardial mass on CCTA $(\mathrm{g})^{*}$ & $49.2 \pm 12.2$ & $39 \pm 13.2$ & $46.5 \pm 15.9$ \\
\hline RV mass on CCTA $(\mathrm{g}) \dagger$ & 0 & 0 & $26.9 \pm 8.6$ \\
\hline LV mass on CCTA (g) & $49.7 \pm 12.7$ & $39.4 \pm 13.4$ & $19.5 \pm 11.1$ \\
\hline \multicolumn{4}{|l|}{ Invasive measurements } \\
\hline FFR & $0.86 \pm 0.06$ & $0.95 \pm 0.03$ & $0.94 \pm 0.05$ \\
\hline FFR Rayflow & $0.81 \pm 0.06$ & $0.92 \pm 0.06$ & $0.91 \pm 0.11$ \\
\hline $\mathrm{Pd}(\mathrm{mm} \mathrm{Hg})$ & $75.3 \pm 14.6$ & $90.1 \pm 20.7$ & $85.6 \pm 17.7$ \\
\hline $\mathrm{Pa}(\mathrm{mm} \mathrm{Hg})$ & $92.6 \pm 16.5$ & $94.1 \pm 21.8$ & $93.7 \pm 16.5$ \\
\hline $\mathrm{Ti}\left({ }^{\circ} \mathrm{C}\right)$ & $-4.2 \pm 1.23$ & $-4.2 \pm 0.95$ & $-3.7 \pm 0.94$ \\
\hline $\mathrm{T}\left({ }^{\circ} \mathrm{C}\right)$ & $-0.55 \pm 0.06$ & $-0.53 \pm 0.19$ & $-0.38 \pm 0.19$ \\
\hline Qnorm (mL/min) & $312.1 \pm 108.8$ & $200 \pm 77.5$ & $226.8 \pm 79.4$ \\
\hline
\end{tabular}

Summary values represent mean \pm SD.

* Myocardial left and right ventricle mass measured from the position of tip of the Rayflow catheter.

tNote: RV mass has been calculated as $20 \%$ of the LV mass.

CCTA, coronary CT angiography; FFR Rayflow, fractional flow reserve during continuous thermodilution (with Rayflow in situ); LAD, left anterior descending; LCX, left circumflex; LV, left ventricle; Pa, central aortic pressure; Pd, distal coronary pressure; Qnorm, normal value of coronary flow in the hypothetical absence of stenosis and infusion catheter; RCA, right coronary artery; RV, right ventricle; T, distal vessel temperature during continuous thermodilution; Ti, infusion temperature.

performed in all 105 coronary arteries. Most patients (54.3\%) had completely normal coronary arteries. The others showed mild wall irregularities in one coronary segment only. No significant adverse events were observed during the invasive coronary angiography and physiological measurements. No chest pain was noted during the infusion of saline. One patient showed transient atrioventricular block during the invasive flow measurement with immediate recovery after stopping saline infusion. This minor side effect is in line with previous data on safety of the invasive flow measurement technique. ${ }^{22} 23$

\section{Relation between CTmass and myocardial perfusion}

Table 2 summarises the absolute values from CT and the invasive

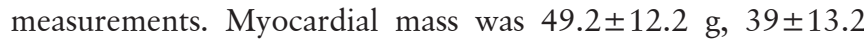
gand $46.5 \pm 15.9 \mathrm{~g}$ for the LAD, LCX and RCA territory, respectively. Total mass equalled $153.3 \pm 33.5 \mathrm{~g}$. Coronary blood flow measured in the LAD was $312.1 \pm 108.8 \mathrm{~mL} / \mathrm{min}$, compared with $200 \pm 77.5 \mathrm{~mL} / \mathrm{min}$ in the LCX and $226.8 \pm 79.4 \mathrm{~mL} / \mathrm{min}$ in the RCA. Total hyperemic blood flow for the complete heart equalled $738.9 \pm 201.9 \mathrm{~mL} / \mathrm{min}$. The percentage difference between relative CTmass per territory and relative flow per coronary artery is presented for the individual patients and per vessel in BlandAltman plots in figure 2. The mean difference was 5.3\% $6.2 \%$ for the LAD territory, $-2.0 \pm 7.4 \%$ for the LCX territory and $-3.1 \pm 3.4 \%$ for the RCA territory. The distributions for mass and flow are visualised for the individual patients in the supplementary file (online supplementary figure S1). Figure 3 shows the relationship between CTmass and invasive flow measurements in the 35 patients. The intraclass correlation between mass and flow is 0.90 in these normal or near-normal patients.

\section{DISCUSSION}

\section{Summary of findings}

The present study shows a close relationship between the mass of the perfusion territory calculated by the specific CT algorithm and invasively measured myocardial perfusion. Therefore, these 

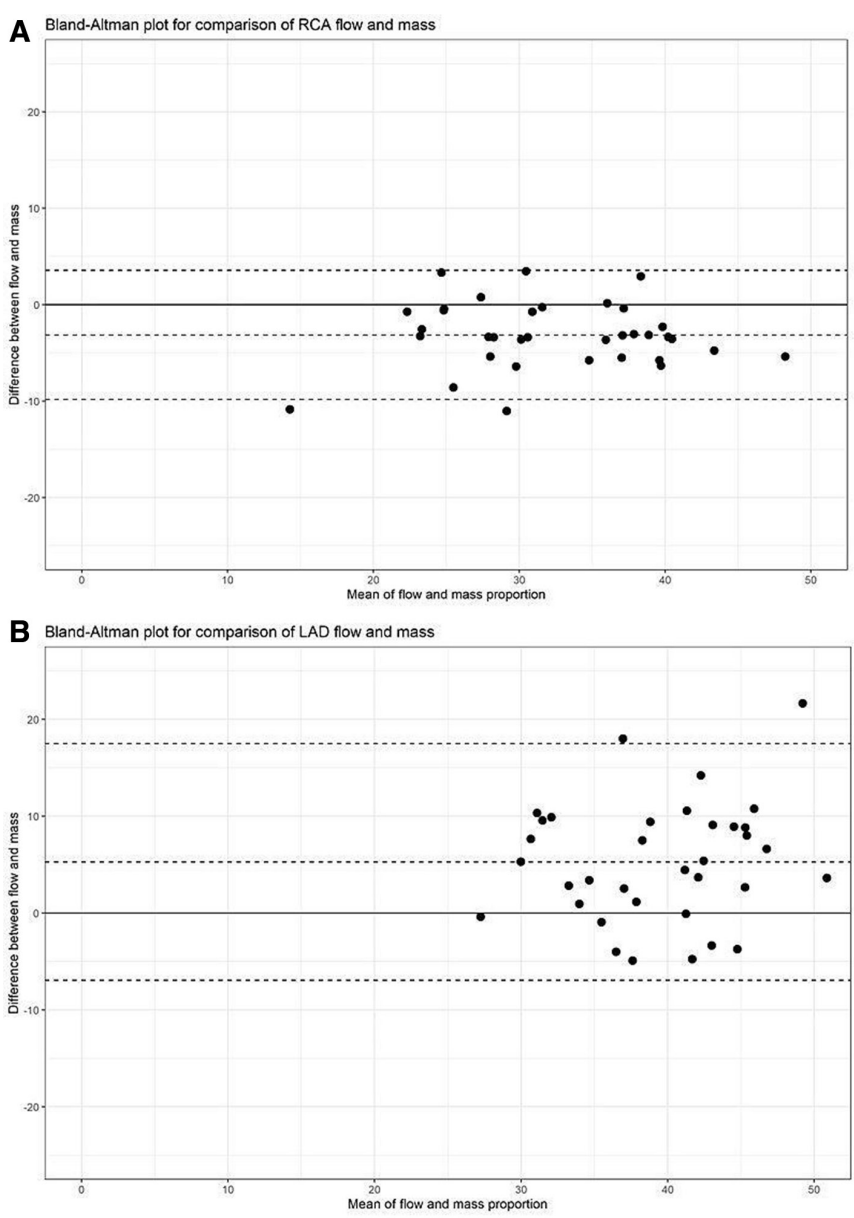

C

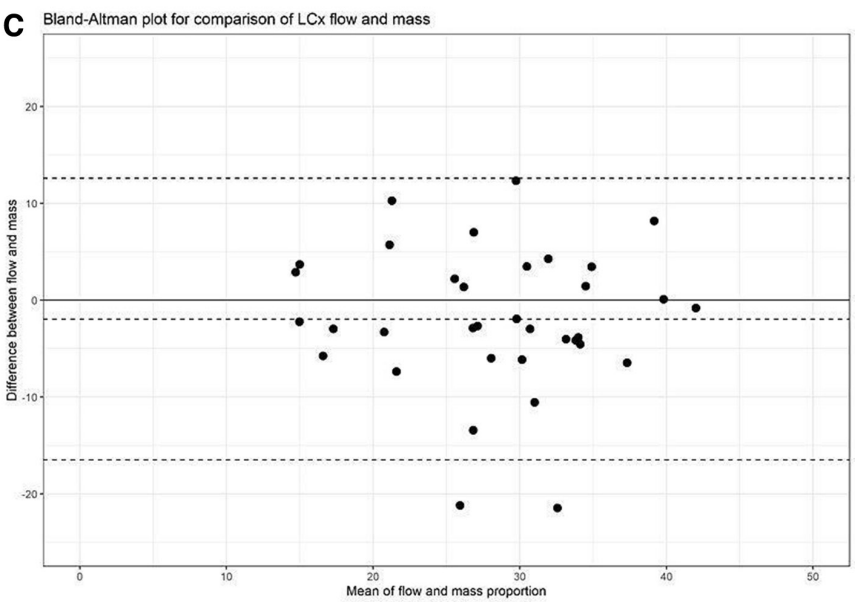

Figure 2 Bland-Altman plots representing CT mass assessment and invasive flow measurement per coronary artery. (A) RCA, (B) LAD and (C) LCx. Dotted line indicates mean value and black solid lines $95 \%$ limits of agreement. LAD, left anterior descending; LCx, left circumflex; $\mathrm{RCA}$, right coronary artery.

data support the reliability of that CT algorithm to estimate myocardial mass at-risk in the proximal coronary arteries and justifies future regional-based CTmass assessment.

\section{Clinical importance of myocardial mass at risk}

Several methodologies are available for assessing myocardial mass or functional stenosis severity separately. But no methodology has been prospectively validated so far for simultaneous assessment of both parameters per territory and within the same

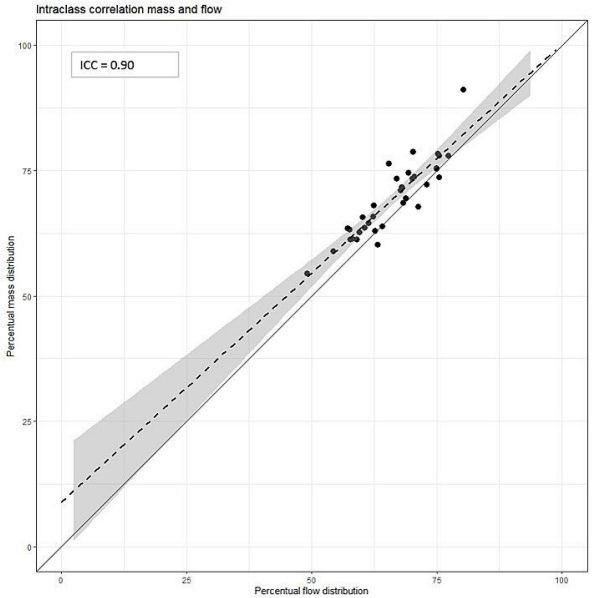

Figure 3 Intraclass correlation (ICC) between mass and coronary blood flow with $95 \% \mathrm{Cl}$ bands.

examination. On one hand, myocardial mass can be assessed non-invasively by several MRI or CT methods. However, no stenosis-specific functional information about the corresponding coronary artery perfusing that territory could be obtained. On the other hand, FFR measured invasively is specific indeed for stenosis-specific ischaemia with excellent spatial resolution. Yet, although FFR for a given stenosis is in itself also determined in part by the extent of the depending viable myocardium, it does not allow to estimate that extent for an individual stenosis. Combining FFR $_{\mathrm{CT}}$ and CTmass within one single non-invasive examination may enable more reliable risk assessment in patients with coronary artery disease. ${ }^{34}$

HeartFlow is already able to accurately assess FFR $_{\mathrm{CT}}$, but mass assumption using their protocol had to be prospectively validated as was done in this study, using invasive measurement of myocardial perfusion as the reference standard.

\section{Myocardial perfusion to mass relationship and comparison with previous studies}

One of the hypotheses of our study is that under physiological circumstances, myocardial perfusion is homogeneous and that myocardial mass is closely linked to myocardial perfusion. This has been extensively investigated in experimental studies, showing proportionality between perfusion and mass. ${ }^{14-16} 24$ This principle is based on the allometric scaling laws by Choy and Kassab ${ }^{14}$ who investigated both the relationship between coronary artery volume and myocardial mass and the relationship between myocardial perfusion and myocardial mass. The latter was the focus in this study and continuous thermodilution was used to measure the myocardial perfusion in millilitres per minute (ie, invasive absolute blood flow measurement). Since there was such a close relationship between the mass derived by HeartFlow and the invasive flow measurements, this confirms the mass calculation.

Murai et $a l^{25}$ did already analyse the relationship between perfusion/flow and mass and compared the Voronoi method with invasive physiology measures (partial myocardial mass (PMM) derived by Doppler flow in a single coronary artery). The difference with our study is that they did not compute total coronary flow neither percentage flow per territory. Our study is an assessment of total myocardial mass and coronary flow of all three coronary arteries. Another difference with our study is that Murai et al validated the PMM method to assess mass and 
our goal is to confirm mass assessment using non-invasive CT imaging. ${ }^{25}$

Further, Ide et al validated the myocardial segmentation algorithm against whole heart histology in 15 pig hearts. ${ }^{6}$ Voronoi segmentation was validated to calculate myocardial mass using CT. Later, it was also validated against SPECT by Kurata et al. ${ }^{5}$ The technique used by HeartFlow differs from Voronoi in that it can account for non-LV feeding vessels using only an LV segmentation as an input. Geometric information about the other heart chambers is not required, which is desirable because it is more difficult to obtain accurate segmentations of the other heart chambers.

All other reported papers ${ }^{10-13}$ are related to volume-to-mass computations, which reported total coronary volume as a ratio to total LV myocardial mass.

\section{Derived flow and mass values compared with literature}

Of interest, both our data for normal hyperemic blood flow and the observed values for myocardial mass are consistent with literature. Normal myocardial LV mass varied between 120 and 160 $\mathrm{g}$ in earlier studies, ${ }^{26}$ whereas in our study total LV mass equalled $110 \mathrm{~g}$. This slightly lower value may be explained by the fact that some proximal side branches in several patients were excluded. The myocardial mass per territory was $49.2 \mathrm{~g}$ for the LAD, $39 \mathrm{~g}$ for the LCX and $46.5 \mathrm{~g}$ for the RCA, and is in line with previous literature. $^{626}$

Total hyperemic coronary blood flow in normal individuals has been well investigated in several human studies and equals $500-600 \mathrm{~mL} / \mathrm{min}$ by PET. ${ }^{27-29}$ In these studies, blood flow to the $\mathrm{RV}$ is not included. Since we also measured flow in the proximal RCA (including perfusion of the RV), this explains the higher values in our study $(738 \pm 201 \mathrm{~mL} / \mathrm{min})$.

\section{LIMITATIONS}

For optimal comparison of flow and mass, in our study, 35 selected patients were included with normal or almost normal arteries as reflected by angiogram, FFR and CT scanning.

For the purpose of this study, this validation in normal patients only is not a real limitation because, once the mass calculation by the CT algorithm has been validated, it can be applied in the analysis of the CT scans even in the presence of coronary artery stenosis. Further studies are mandatory to support this position in patients with extensive coronary artery disease.

Next, we have assumed that our patients were normal or nearly normal by the criteria previously mentioned. Nevertheless, a number of these patients had non-specific chest pain and it cannot be excluded that some of them had microvascular disease, thereby influencing blood flow and the assumption of homogeneous myocardial perfusion.

Only the myocardial volume of the left ventricle was extracted from medical image data and the trabeculae and papillary muscles were excluded. Thus, the total myocardial mass of the left ventricle may be underestimated by this method. The whole septum was included in LV mass calculation. The calculation of the myocardial territories was performed using only the vessels explicitly identified in the CT image data. This could be extended in the future to include synthetic trees generated using branching laws to fill the myocardium. ${ }^{30}$

Furthermore, in our study, homogeneous myocardial density was assumed to be present.

Also, the assumed RV mass was used rather than measured RV mass due to difficulties in resolving the RV wall thickness from CT data. Measuring exact RV mass would have been superior if feasible. The assumption of RV mass being $20 \%$ of the LV mass is a reasonable approximation for patients without pulmonary hypertension. Further, this study shows that the per vessel agreement is high, but we have not investigated segments. Thus, the results can be translated only to ostial lesions, but it remains to be investigated for more distal lesions.

Finally, it should be noticed that this new application of CTmass by HeartFlow is not a currently commercially available outcome of $\mathrm{FFR}_{\mathrm{CT}}$

\section{CONCLUSIONS}

Our study shows a close relationship between the relative mass of the perfusion territory calculated by the specific CT algorithm and invasively measured myocardial perfusion. As such, these data support the use of CTmass to estimate territorial myocardium-at-risk in proximal coronary arteries. However, as indicated in the limitations, further studies in non-selected patients are mandatory to support this position.

\section{Key questions}

\section{What is already known on this subject?}

- It is already known that for a coronary stenosis, both the presence of ischaemia as well as the extent of the perfusion territory distal to the stenosis are clinically relevant. Presence of ischaemia can be reliably assessed by fractional flow reserve (FFR) calculated by $\mathrm{CT}_{\text {angiography }}\left(\mathrm{FFR}_{\mathrm{CT}}\right.$ ). However, assessment of mass by the same set of CT data (CTmass) has not been compared yet with direct invasive measurement of myocardial perfusion.

\section{What might this study add?}

- These data validate regional CTmass, using invasively measured myocardial perfusion as a standard. Outcome of this study supports reliability of that CT algorithm to estimate relative myocardium at risk.

\section{How might this impact on clinical practice?}

- Using the same set of CT data, the presence and severity of proximal coronary artery stenosis and also the extent of myocardium at risk (CTmass) can be assessed simultaneously. Combining FFR $_{\mathrm{CT}}$ and CTmass within one single non-invasive examination may enable more reliable risk assessment in patients with coronary artery disease.

\section{Twitter Jo M Zelis @j_zelis}

Acknowledgements We thank Hyun Jin Kim, Adam Updegrove and Professor Dr Charles Taylor for providing support for FFRCT and percent myocardial blood flow data.

Contributors DCJK: planning, conducting; patient enrolment, data collection and reporting; article writing, submission. SF: conducting; patient enrolment, data collection. MvV: reporting; article writing, data check, statistical check. IC: conducting; patient enrolment, reporting; data collection. JMZ: conducting; patient enrolment, reporting; article check, statistical analysis. MEF: conducting; patient enrolment, reporting; article check. FZ: conducting; patient enrolment, reporting; article check. CC: reporting; data check, statistical check. BDB: reporting; article writing, data check. NHJP: planning; principal investigator, reporting; article writing.

Funding Myomass was an investigator-initiated study supported by an unrestricted educational grant from HeartFlow.

Competing interests SF: institutional consultance/speaker fees from Bayer and Cathworks. CC: grants from HeartFlow, Abbott Vascular, Biosensors, Pie Medical and consultancy fees from HeartFlow and Philips. Member of the advisory board of Abbott Vascular, Pie Medical and Opsens. BDB: institutional grant; Abbott, Boston Scientific, Biotronik AG. Institutional consultance fees; Abbott, Opsens and Boston Scientific outside of the submitted work. Minor equities: Siemens, GE, Bayer, Philips, 
HeartFlow, Edwards Life Sciences and Ceyliad. NHJP: institutional grant; Abbott, Hexacath. Consultant; Abbott, Opsens. Minor equities Philips, GE, ASML, HeartFlow. Consultant GE and personal fees $\mathrm{GE}$.

Patient and public involvement Patients and/or the public were not involved in the design, or conduct, or reporting, or dissemination plans of this research.

Patient consent for publication Not required.

Ethics approval The study was approved by the medical ethics committee of the hospital and all investigators adhered to the principles of the declaration of Helsinki.

Provenance and peer review Not commissioned; externally peer reviewed.

Data availability statement Data are available on reasonable request.

Open access This is an open access article distributed in accordance with the Creative Commons Attribution Non Commercial (CC BY-NC 4.0) license, which permits others to distribute, remix, adapt, build upon this work non-commercially, and license their derivative works on different terms, provided the original work is properly cited, appropriate credit is given, any changes made indicated, and the use is non-commercial. See: http://creativecommons.org/licenses/by-nc/4.0/.

\section{ORCID iDs}

Daniëlle C J Keulards http://orcid.org/0000-0002-0913-4967

Jo M Zelis http://orcid.org/0000-0003-2110-4901

\section{REFERENCES}

1 Hachamovitch R, Hayes SW, Friedman JD, et al. Comparison of the short-term survival benefit associated with revascularization compared with medical therapy in patients with no prior coronary artery disease undergoing stress myocardial perfusion single photon emission computed tomography. Circulation 2003;107:2900-7.

2 Shaw LJ, Iskandrian AE. Prognostic value of gated myocardial perfusion SPECT. J NuCl Cardiol 2004;11:171-85.

3 Toth G, Hamilos M, Pyxaras S, et al. Evolving concepts of angiogram: fractional flow reserve discordances in 4000 coronary stenoses. Eur Heart J 2014;35:2831-8.

4 Ahn J-M, Park D-W, Shin E-S, et al. Fractional flow reserve and cardiac events in coronary artery disease: data from a prospective IRIS-FFR registry (Interventional Cardiology Research Incooperation Society Fractional Flow Reserve). Circulation 2017:135:2241-51.

5 Kurata A, Kono A, Sakamoto T, et al. Quantification of the myocardial area at risk using coronary CT angiography and Voronoi algorithm-based myocardial segmentation. Eur Radiol 2015;25:49-57.

6 Ide S, Sumitsuji S, Yamaguchi O, et al. Cardiac computed tomography-derived myocardial mass at risk using the Voronoi-based segmentation algorithm: a histological validation study. J Cardiovasc Comput Tomogr 2017;11:179-82.

7 Gaur S, Achenbach S, Leipsic J, et al. Rationale and design of the HeartFlowNXT (HeartFlow analysis of coronary blood flow using CT angiography: NeXt sTeps) study. J Cardiovasc Comput Tomogr 2013;7:279-88.

8 Nørgaard BL, Leipsic J, Gaur S, et al. Diagnostic performance of noninvasive fractional flow reserve derived from coronary computed tomography angiography in suspected coronary artery disease. J Am Coll Cardiol 2014;63:1145-55.

9 Taylor CA, Fonte TA, Min JK. Computational fluid dynamics applied to cardiac computed tomography for noninvasive quantification of fractional flow reserve: scientific basis. J Am Coll Cardiol 2013:61:2233-41.

10 Taylor CA, Gaur S, Leipsic J, et al. Effect of the ratio of coronary arterial lumen volume to left ventricle myocardial mass derived from coronary CT angiography on fractional flow reserve. J Cardiovasc Comput Tomogr 2017;11:429-36.
11 Sellers SL, Fonte TA, Grover R, et al. Hypertrophic cardiomyopathy (HCM): new insights into coronary artery remodelling and ischemia from $\mathrm{FFR}_{\mathrm{CT}}$.J Cardiovasc Comput Tomogr 2018;12:467-71.

12 Grover R, Leipsic JA, Mooney J, et al. Coronary lumen volume to myocardial mass ratio in primary microvascular angina. J Cardiovasc Comput Tomogr 2017;11:423-8.

13 Holmes KR, Fonte TA, Weir-McCall J, et al. Impact of sublingual nitroglycerin dosage on $\mathrm{FFR}_{\mathrm{CT}}$ assessment and coronary luminal volume-to-myocardial mass ratio. Eur Radiol 2019;29:6829-36.

14 Choy JS, Kassab GS. Scaling of myocardial mass to flow and morphometry of coronary arteries. J App/ Physiol 2008;104:1281-6.

15 Kassab GS, Finet G. Anatomy and function relation in the coronary tree: from bifurcations to myocardial flow and mass. Eurolntervention 2015;11 Suppl V:V13-17.

16 Seiler C, Kirkeeide RL, Gould KL. Measurement from arteriograms of regional myocardial bed size distal to any point in the coronary vascular tree for assessing anatomic area at risk. J Am Coll Cardiol 1993:21:783-97.

17 Schaap M, van Walsum T, Neefjes L, et al. Robust shape regression for supervised vessel segmentation and its application to coronary segmentation in CTA. IEEE Trans Med Imaging 2011;30:1974-86.

18 Le H, Wong JT, Molloi S. Estimation of regional myocardial mass at risk based on distal arterial lumen volume and length using 3D micro-CT images. Comput Med Imaging Graph 2008;32:488-501.

19 Crystal GJ, Pagel PS. Right ventricular perfusion: physiology and clinical implications. Anesthesiology 2018;128:202-18.

20 van't VM, Adjedj J, Wijnbergen IF, et al. Novel monorail infusion catheter for volumetric coronary blood flow measurement in humans: in vitro validation. Eurolntervention.

21 Aarnoudse W, Van't Veer M, Pijls NHJ, et al. Direct volumetric blood flow measurement in coronary arteries by thermodilution. J Am Coll Cardiol 2007;50:2294-304.

22 Xaplanteris P, Fournier S, Keulards DCJ, et al. Catheter-based measurements of absolute coronary blood flow and microvascular resistance: feasibility, safety, and reproducibility in humans. Circ Cardiovasc Interv 2018;11:e006194.

23 Keulards DCJ, Van 't Veer M, Zelis JM, et al. Safety of absolute coronary flow and microvascular resistance measurements by thermodilution. Eurolntervention 2020. doi:10.4244/EIJ-D-20-00074. [Epub ahead of print: 25 Feb 2020] (Published Online First: 25 February 2020).

24 Kassab GS. Scaling laws of vascular trees: of form and function. Am J Physiol Heart Circ Physiol 2006:290:H894-903.

25 Murai T, van de Hoef TP, van den Boogert TPW, et al. Quantification of myocardial mass subtended by a coronary stenosis using intracoronary physiology. Circ Cardiovasc Interv 2019;12:e007322.

$26 \mathrm{Kim} \mathrm{HY}$, Lim H-S, Doh J-H, et al. Physiological severity of coronary artery stenosis depends on the amount of myocardial mass subtended by the coronary artery. JACC Cardiovasc Interv 2016;9:1548-60

27 Sdringola S, Johnson NP, Kirkeeide RL, et al. Impact of unexpected factors on quantitative myocardial perfusion and coronary flow reserve in young, asymptomatic volunteers. JACC Cardiovasc Imaging 2011:4:402-12.

28 Beanlands RS, Muzik O, Melon P, et al. Noninvasive quantification of regional myocardial flow reserve in patients with coronary atherosclerosis using nitrogen-13 ammonia positron emission tomography. Determination of extent of altered vascular reactivity. J Am Coll Cardiol 1995;26:1465-75.

29 Danad I, Raijmakers PG, Appelman YE, et al. Coronary risk factors and myocardial blood flow in patients evaluated for coronary artery disease: a quantitative [150]H2O PET/CT study. Eur J Nucl Med Mol Imaging 2012:39:102-12.

30 Jaquet $C$, Najman L, Talbot $H$, et al. Generation of patient-specific cardiac vascular networks: a hybrid image-based and synthetic geometric model. IEEE Trans Biomed Eng 2019;66:946-55. 